\title{
PUTRA ACUTE STROKE PROTOCOL
}

Ahmad Sobri Muda ${ }^{1 *}$, Mohd Fandi Al Khafiz Kamis ${ }^{1}$, Mohd Naim Mohd Yaakob ${ }^{1}$, Ezamin Abdul Rahim ${ }^{1}$, Mohamad Khairi Mahmood ${ }^{1}$, Mohamad Syafeeq Faeez Md Noh $^{1}$, Nabila Hanem Arshad ${ }^{1}$, Hasyma Abu Hassan ${ }^{1}$

${ }^{1}$ Department of Radiology, Hospital Pengajar UPM, Faculty of Medicine and Health Sciences, Universiti Putra Malaysia

\section{*Corresponding Author:}

Dr. Ahmad Sobri Muda, Department of Radiology, FPSK, UPM \& Hospital Pengajar UPM, Universiti Putra Malaysia, 43400, Serdang, Selangor. Email: asobri@upm.edu.my

DOI: https://doi.org/10.32896/cvns.v3n4.12-14

Published: 31.12 .2021

Article History: Received September 16, 2021; Revised September 26, 2021; Accepted October 28, 2021

Keywords: Acute Stroke, Magnetic Resonance Imaging, Stroke protocol.

Computed Tomography (CT) is by default the first line neuroimaging method for acute stroke in many institutions; however, early infarct signs on CT can be subtle. Many experiences have proved that magnetic resonance imaging (MRI), especially diffusion weighted imaging (DWI), is significantly more sensitive than $\mathrm{CT}$ in the identification of the infarct core, with better correlation to the final infarct volume (1). Recent scepticism about the current understanding of the ischemic core further supports the need for a more detailed tissue information from imaging in hyperacute stroke (2). MRI provides significantly more valuable information in hyperacute stroke and does not compromise the door to needle (DTN) time (3-5). Sakamoto $Y$ et al have shown that a MRI-first policy is feasible in tertiary general academic teaching hospitals, and significantly reduces the door to reperfusion time (2). Centres adopting the MRI first policy in acute stroke have shown that it is feasible, safe, with satisfactory DTN times (5-7).

Our centre is a newly established teaching hospital and are among few centres which embrace the MRI-first policy for hyperacute stroke. We adopt the Putra Acute Stroke Protocol, with an initial 8-minutes first 3 sequences consisting of DWI, fluid-attenuated inversion recovery (FLAIR) and magnetic resonance angiography (MRA). If DWI shows suspected haemorrhage, the order of the sequences is modified, so that susceptibility weighted imaging (SWI) then comes after DWI (Figure 1). Imaging is paused after the first 3 sequences for the clinical team to determine if intravenous tissue plasminogen activator (IV-tPA) treatment should be administered, or preparations for mechanical thrombectomy (MT) should begin. The order in which subsequent sequences are completed during the scan depend very much on the clinical indication at the time; this usually consists of ASL (arterial spin labelling), SWI (if not included in the first 3 sequences) and MRA of the carotid arteries. Contrasted perfusion MRI and black blood (BB) imaging are performed if contrast is decided to be given.

The adopted protocol enables us to achieve a decision after the third sequence. Aided by the capability to thrombolyse inside the MRI suite, this helps to ensure that the average DTN time is 
less than 60 minutes, as recommended by the majority of current guidelines $(3,4)$.

From January to July 2021, a total of 198 patients underwent the MRI-first policy for hyperacute stroke, adopting the Putra Acute Stroke Protocol. Of these, 40 patients received IVT, while 22 patients underwent MT, representing $20 \%$ and $11 \%$ of the total patients, respectively. Eighty percent of the IVT patients had a DTN time of less than 60 minutes. There were a total of 20 patients who presented with haemorrhage - all of which were identified on the DWI sequence and subsequently confirmed on the SWI sequence. Additionally, the fact that the Putra Acute Stroke Protocol incorporates both SWI and contrasted BB (black blood) imaging enables us to identify haemorrhage, masses, or infection in most instances. Our early observation shows that DWI with ADC is able to identify haemorrhage in acute stroke, even by less experienced radiologists.

From June to December 2020, we had 14 stroke mimics (33\% metabolic causes, 25\% epilepsy, $25 \%$ neuroinfections; the rest due to other causes). Our limited experience showed that all the stroke mimics were accurately diagnosed, thus avoiding inadvertent thrombolysis. Transient ischemic attacks (TIAs) were also clearly identified in our MRI-first policy, avoiding unnecessary treatment. The data is available and is planned to be published but is not yet ready at this juncture.

The DWI and ADC sequences pick-up haemorrhage very well. This is followed by confirmation with the SWI sequence, ensuring that haemorrhagic stroke is not missed when utilizing a MRI-first policy in hyperacute stroke practice. At the moment, we are working on our data of DWI and ADC in identifying haemorrhage in hyperacute stroke. Preliminary findings show an almost $100 \%$ pick-up rate, however, the data is not finalised yet. We hope to publish the findings soon.

There were challenges in establishing a
MRI-first policy in acute stroke, as highlighted by a few studies. In our limited experience, the commitment of the core team to solve initial hurdles, aided by good diagnostic outcomes, strengthens the belief of other stakeholders to embrace MRI for acute stroke. We hope to present these issues in a more comprehensive manner in another study.

Cost and benefit is one of the main issues touched by many people when discussing about MRI services in acute stroke. There is no cost of $\mathrm{x}$-ray tube wear and tear in MRI, therefore, the more cases that are performed should not increase the cost of replacement for parts. The actual cost of MRI is relatively fixed even with examinations performed up to 24 hours a day, 7 days a week. The cost of heat management is also relatively fixed, bar quenching. Many centres leave the MRI machine idle outside of standard working hours, which increases cost inefficiency. A significant percentage of acute strokes present outside of standard working hours, which occupies the idle MRI slots and averages down the MRI overall cost.

The MRI examination market price set previously in our country was set during the early introduction of MRI, reflecting the high purchase price of the MRI scanner then, and the complexity of performing a MRI examination. With price reduction, increased efficiencies, and faster scanning times, a tremendous increase in the capability to perform more examinations per scanner is seen; however, the savings are not reflected well to the price charged per MRI examination - making it appear more costly than it actually is. We are hoping to evaluate, in more detail, the cost versus benefit in future undertakings.

In conclusion, our MRI-first policy for hyperacute stroke adopting the Putra Acute Stroke Protocol did not result in delay of DTN times and did not reduce the adoption for hyperacute stroke treatment, be it either IVT or MT. 


\section{REFERENCES}

1. 1. J.B. Fiebach, MD, P.D. Schellinger, MD, O. Jansen, MD, M. Meyer, P. Wilde, J. Bender, MD, P. Schramm, MD, E. Jüttler, $\mathrm{MD}$, et al. CT and Diffusion-Weighted MR Imaging in Randomized Order DiffusionWeighted Imaging Results in Higher Accuracy and Lower Interpreter Variability in the Diagnosis of Hyperacute Ischemic Stroke. Stroke 2002; 33:2206-2210

2. Mayank Goyal, MD, PhD; Johanna M. Ospel, MD; Bijoy Menon, MD;

Mohammed Almekhlafi, MD; Mahesh Jayaraman, MD; Jens Fiehler, MD; et al. Challenging the Ischemic Core Concept in Acute Ischemic Stroke Imaging. Stroke 2020; 51:3147-3155. DOI:

10.1161/STROKEAHA.120.030620

3. C. Sølling, M. Ashkanian, N. Hjort, C. Gyldensted, G. Andersen, L. Østergaard. Feasibility and logistics of MRI before thrombolytic treatment. Acta Neurol Scand 2009; 120: 143-149 DOI: 10.1111/j.16000404.2008. 01136.x

4. D. Sablot. Results of a 1-year qualityimprovement process to reduce door-to- needle time in acute ischemic stroke with MRI screening. Revue Neurologique.

Volume 173, Issues 1-2, January-February 2017, Pages 47-54

5. D. Sablot et al. Target Door-to-Needle Time for Tissue Plasminogen Activator Treatment with Magnetic Resonance Imaging Screening Can Be Reduced to 45 min. Cerebrovasc Dis 2018; 45: 245-251

6. Noreen Kamal, Elaine Shand, Robert Swanson, Michael D. Hill, Thomas Jeerakathil, Oje Imoukhuede, et al. Reducing Door-to-Needle Times for Ischaemic Stroke to a Median of 30 Minutes at a Community Hospital. Neurol Sci. 2019; 46: 51-56

7. Mal J. Regional Emergency Stroke QuickResponse (RESQ) Network: A Proposed Paradigm of Malaysia Stroke Care Services. Med Health Sci Dec 2020; 16(4): 353-361.

8. Clinical Practice Guideline of Malaysia; Management of Ischaemic stroke. 3rd Edition 2020. CVNS March 2020, 3(1): 1155

\section{Figure 1:}

The Putra Acute Stroke Protocol. The MRI examination will be stopped for IV thrombolysis after the third sequence and administered inside the MRI suite, if indicated. The patient will then be transferred to the angiography suite (after the MRI examination), if indicated for mechanical thrombectomy. If hemorrhage is suspected on the DWI sequence, the SWI sequence will be the sequence that ensues DWI, instead of FLAIR.

\begin{tabular}{|c|c|}
\hline SEQUENCES & ACQUISITION TIME \\
\hline DWI & $01: 47$ \\
\hline FLAIR & $02: 40$ \\
\hline MRA & $04: 09$ \\
\hline SWI & $03: 42$ \\
\hline & \\
\hline PERFUSION IMAGING (CONTRAST) & $02: 05$ \\
\hline BLACK BLOOD VESSEL WALL IMAGING & $05: 00$ \\
\hline OTHER SEQUENCES & \\
\hline
\end{tabular}

